Proceedings

\title{
Eco-friendly Extraction, Optimization and Characterization of Natural Curcuminoids ${ }^{\dagger}$
}

\author{
Nawel Ziani ${ }^{1}$, Toma Nardjes Mouas ${ }^{2, *}$ and Saida Touzouirt ${ }^{3}$ \\ 1 Laboratoire de Chimie-Ingénierie des Matériaux et Nanostructures, Faculté des Sciences, Université Ferhat \\ Abbes Sétif-1, 19000 Sétif, Algeria; zianinouara@gmail.com \\ 2 Laboratoire d'Obtention de Substances Thérapeutiques LOST, Campus Chasbet Ersas, Université frères \\ Mentouri-Constantine 1, 25000 Constantine, Algeria \\ 3 Laboratoire des Ressources Naturelles, Université Mouloud Mammeri, 15000, Tizi-Ouzou, Algeria; \\ tourouirtsaida@gmail.com \\ * Correspondence: mouas.toma.nardjes@umc.edu.dz \\ † Presented at the 1st International Electronic Conference on Biological Diversity, Ecology and Evolution, 15- \\ 31 March 2021; Available online: https://bdee2021.sciforum.net/.
}

Citation: Ziani, N.; Mouas, T.N.;

Touzouirt, S. Eco-friendly

Extraction, Optimization and

Characterization of Natural

Curcuminoids. Proceedings 2021, 68,

x. https://doi.org/10.3390/xxxxx

Publisher's Note: MDPI stays neutral with regard to jurisdictional claims in published maps and institutional affiliations.

Copyright: (c) 2021 by the authors. Submitted for possible open access publication under the terms and conditions of the Creative Commons Attribution (CC BY) license (http://creativecommons.org/licenses/by/4.0/).

\begin{abstract}
The introduction of new species in Algerian biodiversity is a crucial task in our environmental and economical politic, thus some spices of great interest as curcuma, saffron....are introduced by young start-ups as Algerian soil and climate are compatible with its culture; and in the framework of enhancement of such spices, the safe recovery of Curcumin in a keto-enol tautomeric form, a sensitive and versatile pro-oxidant and antioxidant propriety rarely reported, which improve its solubility and promote the effectiveness of Curcumin as natural anticancer agent, is reported. To attend our target, non-thermal and conventional solid-liquid extraction by cold maceration with several solvents' systems and time depending is used, furthermore an optimization using Response Surface Method (RSM) is conducted, then obtained crud is separated using LC chromatography using ethyl acetate/hexane gradient, pure compounds were identified by TLC, MP, UV, IR, and ${ }^{1} \mathrm{H}$ NMR spectroscopy. Experimental and optimized results reveled the best solvent and time: Acetone + shaking $/ 6 \mathrm{~h}$, for extracting maximum yield of $7.52 \%$ and $7.6 \%$ respectively. Besides, obtained Curcuminoids were selectively separated from crud using as supplementary treatment oleoresins solubility in Ether Petroleum propriety, pure Curcumin in tautomeric form was identified, and an improved total yield of purified Curcumin $(2.53 \%)$ is obtained. This eco-friendly, economic and easy process improved Curcuminoids and Curcumin recovery, preservation and efficiency for further industrial applications: building bloc in drug design as anticarcinogen agent, derma-cosmetics, functional foods, nutraceutics, hemysynthesis, and natural dyes which make great profits of introduction of this spicy in Algerian soil and Biodiversity.
\end{abstract}

Keywords: Curcuma longa L. specie; Curcumin; extraction kinetics; RSM; structure analysis

\section{Introduction}

Turmeric was widely used since long centuries in traditional medicine [1,2]. Nowadays, scientific researches proved that the main constituent of Curcuma species "Curcumin" is responsible of the biological activity of turmeric such as anti-inflammatory, antimicrobial, anti-oxidant, anti-parasitic, anti-mutagenic, anti-cancer and anti-cardiovascular diseases [3-6].

These properties have been attracting for a long time the interest of various scientists, since this natural product with multi-targeted effects have less adverse effects than synthetic drugs with non observed toxicity even when taken at very high dose over long time $[7,8]$, however these therapeutically effects are limited by a luck of solubility due to its diketo chemical structure, and one way of recent researches for improving its physicochemical proprieties and reactivity, is structural modulation and transformation, another 
way is to find an appropriate medium to generate a keto-enol tautomeric form and stabilize it.

Therefore, to meet industry demands for these biobased compounds with high quality, extraction is by far the most important stage in the recovery process, and depending on different factors such as high productivity, integrity and selectivity towards the target compound must be considered [9]. Extraction efficiency also depends on the localization and nature of the polyphenols. Conventional solvent extraction is the most widespread technique used at laboratory and at industrial scale to extract bioactive compounds from plant matrix, among which cold maceration presents the advantage of increasing mass transfer process with lower energy consumption and higher physical properties, in addition to sensible and volatile food compounds preservation.

In this context, the present study report an economic and Eco- friendly extraction of natural curcuminoids by cold maceration using: solvents' polarity, physical proprieties (shaking) and time depending, Insilco optimization of this method using RSM is also given. Furthermore, a selective extraction, isolation, purification and identification of main curcuminoids are investigated to prove the efficiency of the optimized matrix in the recovery of these prized bioactive compound in the target keto-enol form to reach and assess its optimal therapeutically effect and increase its exploitation possibility.

\section{Experiments}

\subsection{Plant Materials}

Turmeric rhizomes were commercially purchased from a local traditional shop, cleaned, dried and sprayed into a powder with an electrical pulvirisator until content was about $20 \mathrm{~g}$ dry weight. All other solvents and reagents used in this work were of analytical grade and purchased from Sigma-Aldrich Company Ltd. (Germany).

\subsection{Extraction}

Solvent extraction was carried out using cold maceration solid-liquid technique, where different organic solvents' polarity and extraction times were used to obtain optimum conditions.

Briefly, it consisted into the extraction of the hole crud of rhizomes $(20 \mathrm{~g})$ with $70 \mathrm{~mL}$ of Ethanol, Chloroform, Dichloromethane, Ethyl Acetate, Acetone and Acetone with shaking namely S1, S2, S3, S4, S5, S6 respectively, under magnetic agitation for 3, 4, 5, $6 \mathrm{~h}$, then filtered with a Büchner leading to 24 crud extracts, the filtrates were evaporated to concentrates using rotary evaporation at $40{ }^{\circ} \mathrm{C}$ and stored in an amber coated bottle at $4{ }^{\circ} \mathrm{C}$. The extraction yield was calculated as follows:

$$
y=m_{1} / m_{2} \times 100
$$

where $y$ is the yield of dried extract, $m_{1}$ is the mass of dried extract; $m_{2}$ is the mass of the sample.

\subsection{Optimization of Curcuminoids Extraction by Response Surfaces Method (RSM)}

One of the objectives of this study is the optimization of the extraction yield of Curcuminoids using solid-liquid extraction by cold maceration in order to maximize the recovery of these bioactive compounds for industrial purpose. The optimal conditions are determined using central composite designs (CCD) [10]. Runs were performed by varying the extraction time and solvents' systems. The ratio of the mass/volume was considered constant during the experiments.

\subsection{Central Composite Design}

In this study, the central composite design consists of two-level factors. The extraction yield of curcuminoids represents the response $\mathrm{Y}$ that is closely related to the extrac- 
tion time and solvents. Extraction time is proportional to the extraction yield; it is a quantitative variable and considered as an important factor because it directly affects the yield. The second qualitative variable is solvents' systems, chosen because to their influence on extraction selecting of bioactive compounds and increasing yield. The experiment matrix as well as the statistical analysis of the results was made using MODDE software, version 6 [11].

The number of trials $\mathrm{N}$ is taken from full factorial design $(\mathrm{NF}=2 \mathrm{k})$. Three additional points were added to the center of the domain (N0) in order to evaluate the reproducibility of the response and the experimental error and others points "Axial" for the quantitative factor.

$$
\mathrm{N}=\mathrm{NF}+\mathrm{N} \alpha+\mathrm{N} 0 ; \mathrm{N}=24 \text { experiences }
$$

The Equation (1) is given as follows

$$
Y=a_{0}+a_{1} X_{1}+a_{2} X_{2}+a_{12} X_{1} X_{2}+a_{11} X_{1}^{2}+a_{22} X_{2}^{2}+e
$$

$Y$ represents the yield of crud curcuminoids;

$X_{1}$ and $X_{2}$ are the coded variables respectively for the extraction time and the solvents' system; aij: are the coefficients of the model $(i=0,1,2$ and $j=1,2)$

\section{Isolation of Curcuminoids}

\subsection{Ether Petroleum Treatment}

A second step using Petroleum Ether came to extract more selectively curcuminoids from the best extract yield and separate them from oleoresins which are more soluble in Petroleum Ether. For this, obtained crud was mixed three times with $15 \mathrm{~mL}$ of Ether Petroleum. Following extraction, the remaining solvent was removed using rotary evaporation at $40{ }^{\circ} \mathrm{C}$ and stored in an amber coated bottle at $4{ }^{\circ} \mathrm{C}$. The extraction yield was calculated as follows:

$$
y=m_{1} / m_{2} \times 100
$$

where $y$ is the yield of dried extract, $m_{1}$ is the mass of dried extract, $m_{2}$ is the mass of the Sample.

\subsection{Thin Layer Chromatography}

A rapid screening of suitable solvent system for isolating maximum compounds of turmeric rhizomes extract was done using thin layer chromatography (TLC). A chromatographic tank was filled with several solvents' systems to screen which is the best for curcuminoids resolution, and kept covered for $10 \mathrm{~min}$ to get saturated with solvent's vapors. Silica Gel 60 F254 precoated aluminum TLC plate $10 \times 2 \mathrm{~cm}$ (Merck, Germany) was used as a stationary phase. The crude concentrated turmeric rhizomes extract was loaded on the TLC plate and put in the tank containing solvents. Physic revelation of spots was done using U.V lamp at 254 and $365 \mathrm{~nm}, \mathrm{R} f$ values were then calculated according to Touchstone [12].

\subsection{Column Chromatography}

Isolation of curcuminoids from Turmeric was done through column chromatography using the mobile phase selected on the basis of TLC screening. A glass column $(100 \times 3 \mathrm{~cm})$ was filled with silica gel powder Kieselgel Merck (60-120 mesh), and was then loaded with hexane. Afterwards, concentrate crude extract was added on top with a pipette. The compounds of rhizomes extract were isolated using a gradient of $0,2.5,5,10,20,30,40$, $50,60,70,80,90$, and $100 \%$ ethyl acetate in hexane as mobile phase, with a drip elution rate. Afterward, these isolated fractions were analyzed through TLC again. The isolated fractions containing the same compounds based on $R f$ values were pooled together, and 
fractions having a single spot on TLC plates were subjected to characterization and identification analysis.

\subsection{Identification and Characterization}

- Fourier transform infrared spectroscopy (FT-IR) analysis

For Infrared (IR) spectra of isolated compounds, $1 \mathrm{mg}$ of powder was mixed with $\mathrm{KBr}$ to form a plate. Absorption peaks of functional groups were recorded using Fourier transform spectrometry instrument SHIMADZU HYPER, FTIR-8201 PC in the range of $4000-400 \mathrm{~cm}^{-1}$.

- $\quad$ UV-VIS spectroscopy

UV-Visible absorbance was performed in scan mode with a SHIMADZU UV 16A spectrophotometer.

- $\quad$ Melting point

The melting points of isolated compounds were recoded on a digital apparatus type STUART.

- $\quad$ Nuclear magnetic resonance spectroscopy ( $\left.{ }^{1} \mathrm{H}-\mathrm{NMR}\right)$

1D-1H-nuclear magnetic resonance (NMR) spectrum of the isolated and purified compounds was performed using NMR (Bruker Avance), $400 \mathrm{MHz}$, in $\mathrm{CdCl}_{3}, 1 \mathrm{H}$ chemicals shifts $(\delta)$ are given in ppm (the residual peak of deuterated solvent was used as reference).

\subsection{Statistical Analysis}

Each experiment was performed in five replicates and the data was subjected to calculations of mean \pm S.E. The mean values were used for drawing the graphs.

\section{Results and Discussion}

\subsection{Extraction Solvents Screening and Extraction Yields}

According to obtained results we can observe that acetone with shaking (S6) exhibit the best extract yield from the first recorded hours $(3 \mathrm{~h})$ till the end $(6 \mathrm{~h})$ with $7.52 \%$ of yield extraction, this exhibit the role of shaking process in the efficiency of the recovery of target compounds, this could be explained by its facilitation of curcuminoids mass transfer from the matrix to the solvent, in fact conventional methods operate through cell permeation followed by solubilizing the active constituents by the extracting solvent. Curcumin present inside the oleoresin cells which in turn is covered by tightly packed cork cells probably makes the entry route for the solvent tougher [13], so pumice stone shaking process probably facilitates entry of the extracting solvent to solubilize out the target compound, thus lead to faster and efficient extraction.

Furthermore, the choice of Acetone solvent to get better extraction yield, is in line with previous studies that reports acetone as better solubilizing and extraction solvent than ethanol for Curcuminoids in conventional methods as well as in non-conventional methods [14], the two factors combination in one system S6 increased extract yield in shorter time to maximize the recovery of target compounds with more selectivity, efficiency and quality (stability).

\subsection{Optimization by RSM}

\subsubsection{Statistical Study of Extraction Results}

The obtained results by central composite design gave the best yield for acetone/shaking system, with $7.52 \%$ yield during $6 \mathrm{~h}$ of extraction.

\subsubsection{Validation of the Mathematical Model}

The validity of the mathematical model was done using MODDE 6 software. 


\subsubsection{Adequacy of the Model}

The experimental values are nearly close to those predicted. The coefficients values of R2 and Q2 are 0.976 and 0.950 , respectively. These values are close to equal, so the model is adequate.

\subsubsection{Significant Factors}

According to significance level of 5\%, all solvents except S1 (ethanol) are significant, among which S3 (dichloromethane), S4 ethyl acetate), and S5 (acetone) influence negatively, while S2 (chloroform) and S6 (acetone with shaking) influence positively the extract yield.

Consequently, only time, solvent S2 (chloroform) and S6 (acetone with shaking) are considered as significant factors for the extraction and positively influence extraction yields.

\subsubsection{Analysis of Variance}

The model is considered valid when the experimental Fisher F (Fobs) is greater than tab Fisher F (Fcrit); the $p$-value is close to zero. Therefore, the model is statistically significant and should be suitable for given response.

\subsubsection{Residues Study}

Evolution of residues with the studied factors, shows random dispersion of residues.

Thus, it indicates that the (acetone/shaking) system extraction during 6hours is the most suitable to get the best possible extraction yield.

\subsection{Optimization}

The optimum yield was obtained for (acetone/shaking) with $7.6 \%$ yield during $6 \mathrm{~h}$ of extraction.

\section{Isolation of Curcumin from Turmeric Rhizome Extract}

Rhizomes" extract (395 mg/1.98\% yield) obtained after selective extraction with S6 and ether petroleum treatment, was subjected to isolation of bioactive compounds through TLC and column chromatography. Hexane: ethyl acetate (70:30; v:v) solvent system gave a good resolution for isolating curcuminoids on TLC plate. The gradient solvent system used in column chromatography isolation lead to elute a total of 9 fractions of 50 mL each. Only F3, F4, F5 gave a single, yellow colored spot, whereas the other fractions gave multiple spots, $\mathrm{F} 4$ with a $\mathrm{R} f$ value of 0.78 corresponding to the main standard compound Curcumin, was obtained with $2.53 \%$ of yield better than the conventional Soxhlet assisted acetone after $8 \mathrm{~h}$ of extraction resulted in a 2.1\% Curcumin yield [14], is consequently chosen for further characterization and validation using $1 \mathrm{H}-\mathrm{NMR}, \mathrm{FT}-\mathrm{IR}$, melting point and UV-VIS analysis.

\subsection{Identification and Characterization of Purified Curcumin through 1HNMR, FT-IR, Melting} Point and UV-VIS

The $1 \mathrm{H}-\mathrm{NMR}$ spectrum of the isolated compound $\mathrm{F} 4$ exhibit six peaks regions: $\mathrm{C}, \mathrm{H}$, D, E, F, G [15-17].characteristic of Curcumin functions, Therefore, the isolated compound F4 was identified as [1,7-bis-(4-hydroxy-3-methoxyphenyl)-1,6-hepta diene-3,5-dione].

Although, Curcumin is commonly named as 1,7-bis (4-hydroxy-3-méthoxyphényl) 1,6-hépatadiène3,5-dione, highlighting its dikitone tautomeric form, however, few studies reported also two other possible rare forms asymmetric kito-enol tautomers attributed to its interactions with acid ph medium and solvents like CDCl3 [17,18].

Indeed, apparition of a doublet in region I (6.4-6.5 ppm) indicating the presence of non equivalent proton and the appearance of a Hhydroxyl at $4.1 \mathrm{ppm}$ suggests the kito-enol forms of Curcumin. 
The importance of the tautomerism of Curcumin has been examined in a study investigating the molecular mechanism of the observed synergy between Curcumin and water soluble antioxidants in cancer chemoprevention at physical conditions [19]. It has been observed that the Curcumin radical preferentially exists as a phenoxyl-type species, which is more hydrophilic than the keto form. Being more hydrophilic, it is preferentially moved to the external side of the cell membrane; this effect is probably responsible of Curcumin effectiveness as a scavenger of carcinogenic free radicals [20].

- $\quad$ The FT-IR spectra of isolated F4 corresponding to the main standard Curcumin revealed characteristic absorption peaks in $\mathrm{KBr}$ pallets at $1510 \mathrm{~cm}^{-1}(\mathrm{C}=\mathrm{O}), 3500 \mathrm{~cm}^{-1}$ (OH aromatics function), $1596 \mathrm{~cm}^{-1}$ (C=C aromatics), $1029 \mathrm{~cm}^{-1}$ (C-O-C) et 1278 (C-O enol) [21,22].

- $\quad$ Melting point

F4 Melting point was observed at $183.5^{\circ} \mathrm{C}$, which indicate the purity of obtained Curcumin.

- UV-VIS

UV-VIS spectra of isolated Curcumin (F4) was performed in ethyl acetate solution and recorded in the region of 800-200 $\mathrm{nm}$ at room temperature, it reveled two absorption bands around $417 \mathrm{~nm}$ and $263 \mathrm{~nm}$, corresponding to $\pi \rightarrow \pi^{*}$ and $n \rightarrow \pi^{*}$ transitions respectively, while it shows one absorption at $429 \mathrm{~nm}$ in methanol, which is in nice agreement with [23].

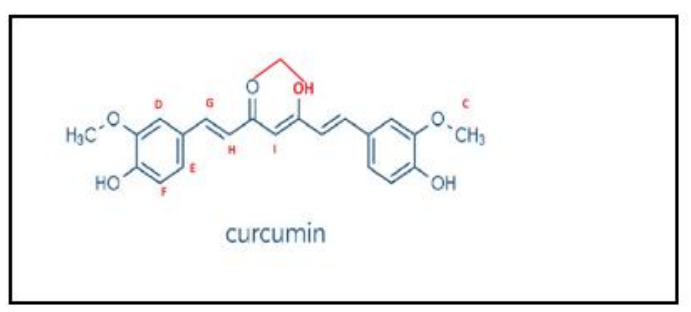

Figure 1. Tautomeric form of Curcumin.

\section{Conclusions}

Conventional methods still interesting extracting methods prized at industrial scale, because it is known to be safe, economic, non-thermal and easy extraction method; besides, when optimized using several solvent systems and time in addition to a supplementary treatment, it could be very interesting method to maximize the recovery of thermofragile natural biomolecules particularly Curcumin from turmeric rhizomes and its structure stability preservation as building bloc for industrial purpose. The present study highlighted the role of solvent medium in the selectivity and structural stability of curcuminoids using polarity proprieties in extraction, mechanic propriety of shaking and time as decisive factors in improving the recovery of target bioactive compounds. In fact, shaking extractions under optimized process control using experimental design methodology through central composite design exhibit nearly the same result for predicted and experimental yields namely acetone with pumice stone during $6 \mathrm{~h}$ to have $7.6 \%$ crud which is more than most reported results in literature. Furthermore, this selective extraction and treatment lead also to maximize pure Curcumin recovery $(2.52 \%)$, isolated Curcumin was identified through TLC, melting point, IR, UV and NMR $1 \mathrm{H}$ spectroscopy which reveled the target keto-enol tautomery, a very fragile and rare form, giving a versatile propriety to Curcumin reactivity as pro-oxidant and antioxidant at the same time; this effect is probably responsible of Curcumin effectiveness as a scavenger of carcinogenic free radicals, promoting it as anticancer agent for further applications. 
Author Contributions: T.N.M. conceived and designed the experiments, analyzed the data and wrote the paper; N.Z. and S.T. performed the experiments; analyzed the data. All authors have read and agreed to the published version of the manuscript.

Institutional Review Board Statement: Not applicable.

Informed Consent Statement: Not applicable.

Data Availability Statement: Not applicable.

Acknowledgments: Authors would like to thank Algerian Ministry of Higher Education and Scientific Research DGEFS, and the Algerian Directorate General for Scientific Research and Technological Development DGRSDT for financial fund.

Conflicts of Interest: The authors declare no conflict of interest. The founding sponsors had no role in the design of the study; in the collection, analyses, or interpretation of data; in the writing of the manuscript, and in the decision to publish the results.

\section{References}

1. Gupta, S.C.; Kismali, G.; Aggarwal, B.B. Curcumin, a component of turmeric: From farm to pharmacy. Biofactors 2013, 39, 2-13.

2. Ouelbani, R.; Bensari, S.; Mouas, T.N.; Khelifi, D. Ethnobotanical investigations on plants used in folk medicine in the regions of Constantine and Mila (North-East of Algeria). J. Ethnopharmacol. 2016, 194, doi:10.1016/j.jep.2016.08.016.

3. Ahsan, H.; Parween, N.; Khan, N.U.; Hadi, S.M. Pro-oxidant, anti-oxidant, and cleavage activities on DNA of curcumin and its derivatives demethoxycurcumin and bisdemethoxycurcumin. Chem. Biol. Interact. 1999, 121, 161-175.

4. Peret-Almeida, L.; Cherubino, A.P.F.; Alves, R.J.; Dufosse, L.; Gloria, B.A.M. Separation and determination of the physicochemical characteristics of curcumin, demethoxycurcumin and bisdemethoxycurcumin. Food Res. Int. 2005, 38, 1039-1044.

5. Çıkrıkçı, S.; Mozioğlu, E.; Yılmaz, H. Biological activity of curcuminoids isolated from Curcuma longa. Rec. Nat. Prod. 2008, 2, 19-24.

6. Campbell, M.S.; Fleenor, B.S.The emerging role of curcumin for improving vascular dysfunction: A review. Crit. Rev. Food Sci. Nutr. 2017, 58, 2790-2799.

7. Li, H.; Sureda, A.; Devkota, H.P.; Pittalà, V.; Barreca, D.; Silva, A.S.; Tewari, D.; Xu, S. Seyed Mohammad Nabavi, Curcumin, the golden spice in treating cardiovascular diseases. Biotechnol. Adv. 2020, 38, 107343; ISSN 0734-9750, doi:10.1016/j.biotechadv.2019.01.010.

8. Lao, C.D.; Ruffin, M.T.; Mack, T.; Normolle, D.; Heath, D.D.; Murray, S.I.; Bailey, J.M.; Boggs, M.E.; Crowell, J.; Rock, C.L.; et al. Dose escalation of a curcuminoid formulation. BMC Complement. Altern. Med. 2006, 6, 10.

9. Osorio-Tobón, J.F.; Carvalho, P.I.; Rostagno, M.A.; Petenate, A.J.; Meireles, M.A.A. Extraction of curcuminoids from deflavored turmeric (Curcuma longa L.) using pressurized liquids: Process integration and economic evaluation. J. Supercrit. Fluids 2014, 95, 167-174.

10. Goupy, J.; Creighton, L. Introduction Aux Plans D'expériences, 3rd ed.; DUNOD: Paris, France, 2006; p. 336.

11. Software, M. Umetrics AB. 2001.

12. Touchstone, J.C. Practice of Thin Layer Chromatography, 3rd ed.; John Wiley \& Sons: Hoboken, NJ, USA, 1992; 400p.; ISBN $0471612227 / 9780471612223$.

13. Mandal, V.; Mohan, Y.; Hemalatha, S. Microwave assisted extraction of curcumin by sample-solvent dual heating mechanism using Taguchi L9 orthogonal design. J. Pharm. Biomed. Anal. 2008, 46, 322-327, doi:10.1016/j.jpba.2007.10.020.

14. Wakte, P.S.; Sachin, B.S.; Patil, A.A.; Mohato, D.M.; Band, T.H.; Shinde, D.B. Optimization of microwave, ultra-sonic and supercritical carbon dioxide assisted extraction techniques for curcumin from Curcuma longa. Sep. Purif. Technol. 2011, 79, 50-55, doi:10.1016/j.seppur.2011.03.010.

15. Nagahama, K.; Utsumi, T.; Kumano, T.; Maekawa, S.;Oyama, N.; Kawakami, J. Discovery of a new function of curcumin which enhances its anticancer therapeutic potency. Sci. Rep. 2016, 6, 30962, doi:10.1038/srep30962.

16. Sarika, P.R.; Nirmala, R.J. Curcumin loaded gum arabic aldehyde-gelatin nanogels for breast cancer therapy. Mater. Sci. Eng. C 2016, 65, 331-337, doi:10.1016/j.msec.2016.04.044.

17. Payton, F.; Sandusky, P.; Alworth, W.L. NMR Study of the Solution Structure of Curcumin. J. Nat. Prod. 2007, 70, 143-146, doi:10.1021/np060263s.

18. Roughley, P.J.; Whiting, D.A. Experiments in the biosynthesis of curcumin. J. Chem. Soc. Perkin Trans. 1973, 1, 2379, doi:10.1039/p19730002379.

19. Jovanovic, S.V.; Boone, C.W.; Steenken, S.; Trinoga, M.; Kaskey, R.B. How curcumin works preferentially with water soluble antioxidants. J. Am. Chem. Soc. 2001, 123, 3064-3068.

20. Euterpio, M.A.; Cavaliere, C.; Capriotti, A.L.; Crescenzi, C. Extending the applicability of pressurized hot water extraction to compounds exhibiting limited water solubility by $\mathrm{pH}$ control: Curcumin from the turmeric rhizome. Anal. Bioanal. Chem. 2011, 401, 2977-2985, doi:10.1007/s00216-011-5383-7.

21. Mohan, P.R.K.; Sreelakshmi, G.; Muraleedharan, C.V.; Joseph, R. Water soluble complexes of curcumin with cyclodextrins: Characterization by FT-Raman spectroscopy. Vib. Spectrosc. 2012, 62, 77-84, doi:10.1016/j.vibspec.2012.05.002. 
22. González-Albadalejo, J.; Sanz, D.; Claramunt, R.M.; Lavandera, J.L.; Alkorta, I.; Elguero, J. Curcumin and curcuminoids: Chemistry, structural studies and biologicalproperties. An. Real Acad. Farm. 2015, 81, 278-310.

23. Goel, A.; Kunnumakkara, A.B.; Aggarwal, B.B. Curcumin as "Curecumin": From kitchen to clinic. Biochem. Pharmacol. 2008, 75, 787-809, doi:10.1016/j.bcp.2007.08.016. 